\title{
Schwerpunkt „Nephrologie/Hypertensiologie“ Im Fokus: die resistente Hypertonie
}

\begin{abstract}
In Im vorliegenden Schwerpunktheft Nephrologie/Hy pertensiologie präsentieren wir aktuelle Themen dieser Fachgebiete. Die resistente Hypertonie steht gegenwärtig nicht nur wegen ihrer großen praktischen Bedeutung im Fokus des Interesses, sondern auch, weil derzeit eine Reihe neuer Verfahren möglicherweise das bewährte Vorgehen bei therapieresistentem Bluthochdruck ergänzen kann. Eine resistente Hypertonie liegt definitionsgemäß vor, wenn der Zielblutdruck trotz einer adäquat dosierten antihypertensiven Dreierkombination - unter Einschluss eines Diuretikums - nicht erreicht wird. Von einem therapieresistenten Hochdruck ist eine unkontrollierte Hypertonie zu unterscheiden. Bei Letzterer wird das Blutdruckziel verfehlt, weil der Patient keine ausreichende Medikation im Sinne
\end{abstract} deroben genannten Definition erhal„Es ist wichtig, eine resistente Hypertonie von einem unkontrollierten Hochdruck zu unterscheiden." ten respektive eingenommen hat. Der Beitrag auf S. 34 ff. erläutert diese Begriffe und gibt den derzeitigen Stand der Diagnostik und Therapie bei resistenter Hypertonie wieder. Auch die therapeutischen Optionen bei resistenter Hypertonie werden ausführlich diskutiert: Zwar sind für therapieresistente Hypertoniker diverse Reserveantihypertensiva wie Alphablocker, zentral wirksame Sympathikolytika, Aldosteronantagonisten oder Vasodilatatoren verfügbar. Allerdings sind diese Reservemedikamente häufig nur mäßig effektiv und können je nach Substanzgruppe eine Vielzahl von Nebenwirkungen induzieren. Weil aber ein resistenter Bluthochdruck das kardiovaskuläre Risiko der Betroffenen vervielfacht, ist die Weiterentwicklung der antihypertensiven Therapie dringend notwendig. Eine möglicherweise vielversprechende Option stellen die Endothelinantagonisten dar. Mit dem gegenwärtigen Stellenwert dieser gefäßaktiven Substanzen beschäftigt sich ein weiterer Beitrag (S. 37f.). Bislang vereiteln die Nebenwirkungen den breiten klinischen Einsatz der Endothelinantagonisten.
Aber nicht nur neue, sondern auch alte und bewährte Medikamente geben Anlass zur Diskussion. Diuretika gehören zu den ältesten Antihypertensiva. Speziell das Hydrochlorothiazid (HCT) ist nicht nur weit verbreitet, sondern auch als effektives Antihypertensivum sehr erfolgreich einsetzbar. Dennoch gibt es interessante Hinweise dafür, dass wir bei der Diuretikamedikation unserer Hochdruckpatienten in Zukunft variabler vorgehen sollten. Die "Konkurrenten" des Hydrochlorothiazids, das Chlorthalidon und das Indapamid, können die Hypertoniebehandlung ergän„Diuretika sollten in der Behandlung von Hypertoniepatienten künftig variabler eingesetzt werden." zen und bereichern, wie der Bei-

trag aufS. 39 f. zeigt.

Schließlich werden anhand einer interessanten Kasuistik (S. 41 ff.) die konservativen und operativen Therapiemöglichkeiten bei der retroperitonealen Fibrose (Morbus Ormond) diskutiert. Die Erkrankung zählt zu den selteneren Ursachen einer postrenal bedingten Niereninsuffizienz. Die chronisch verlaufende unspezifische Entzündung des Retroperitonealraums mit konsekutivem fibrotischem Umbau führt im Krankheitsverlauf zu einer Ummauerung und/oder Kompression von Leitungsstrukturen. Eine frühe Diagnose ist aufgrund der unspezifischen Symptome schwierig. Oft ist zum Zeitpunkt der Diagnosestellung bereits die Nierenfunktion eingeschränkt. Eine zügige Therapie mit Steroiden und/oder Immunsuppressiva kann den Progress und insbesondere den irreversiblen Nierenfunktionsverlust verhindern.

Wir wünschen unseren Lesern viel Spaß beim Lesen dieses Überblicks über aktuelle Probleme in der Nephrologie und Hypertensiologie.

Ihre

Joachim Hoyer und Walter Zidek 